\title{
Etude des laves torrentielles sur modèle réduit en similitude des phénomènes naturels
}

\author{
Philippe Coussot et Dominique Laigle \\ CEMAGREF, Division Protection contre les Erosions, \\ Domaine Universitaire, B.P. 76, 38402 St-Martin-d'Hères, France \\ Tél : 76762766
}

Les laves torrentielles sont des mélanges boueux et rocailleux qui surviennent dans les torrents de montagne après de fortes ou longues pluies et peuvent causer d'importants dégâts. Ces phénomènes sont $a$ priori complexes. Il s'agit de coulées formées d'un mélange d'eau, d'argile, de sable, de cailloux et de gros blocs (jusqu'à plusieurs mètres de diamètre) qui ont un caractère transitoire marqué. Par ailleurs de nombreux phénomènes particuliers viennent compliquer la compréhension et la prédiction du phénomène d'ensemble : l'hétérogénéité du matériau, les dépôts et reprises, l'érosion, les apports d'eau, la migration des blocs, la formation d'un front constitué essentiellement de gros blocs, les éventuels glissements ou fracturations du matériau au cours du mouvement. Enfin les conditions aux limites géométriques des écoulements sont complexes: pente, section et rugosité variables, extensions latérales possibles sur le cône de déjection. Tenant compte des observations de terrain [1] nous avons choisi d'aborder le problème de ces écoulements par la mécanique des fluides [2]. Nous nous sommes donc affranchis d'un grand nombre de phénomènes particuliers que nous avons supposés négligeables dans un premier temps. Même dans ces conditions, prédire correctement les conséquences de l'écoulement naturel d'un fluide à seuil à l'aide des modèles numériques existants reste une tâche difficile et aventureuse car ceux-ci n'ont pas encore été suffisamment validés. Les études sur modèle réduit peuvent donc être d'une grande utilité pour des applications pratiques. Encore faut-il être capable de réaliser de telles études de manière pertinente, c'est-à-dire, dans le cas d'un modèle réduit, en respectant une similitude correcte entre l'écoulement naturel et l'écoulement réduit. L'objet de cet article est d'examiner les possibilités actuelles pour la réalisation de ce type de modèle. On notera qu'il ne s'agit pas a priori de réaliser un modèle réduit pour étudier plus précisément les phénomènes physiques mais pour résoudre des problèmes concrets.

La reproduction de laves torrentielles sur modèle réduit n'a jusqu'à présent pratiquement pas fait l'objet de travaux scientifiques. A notre connaissance les seuls travaux de ce type ont été réalisés par la Sogreah [3-4]. Le principe adopté alors pour respecter une certaine similitude entre la réalité et le modèle consistait à fabriquer un matériau qui, relâché brutalement de l'amont, avait, sur le modèle, une allure générale (bourrelets latéraux) ou des caractéristiques géométriques et cinématiques (en similitude) identiques à celles observées lors d'un écoulement particulier sur le site naturel. Les autres travaux qui ont eu pour but d'étudier expérimentalement des écoulements à surface libre de laves torrentielles ne se sont pas appuyés sur des règles de similitude, ils ont seulement été réalisés en utilisant des matériaux "analogues » [5-7]. Grâce aux développements récents concernant la rhéologie et les lois d'écoulement des laves torrentielles $[2,8]$ il nous semble maintenant possible de définir quelques bases plus solides pour l'étude des laves torrentielles sur modèle réduit.

Dans la première partie, prenant le parti de réaliser une

\section{Debris flow small-scale study in similarity with natural events}

Small-scale study of complex debris flows are useful to solve many practical problems. It is hard to carry out a complete similarity between small-scale flows and natural events. Indeed the material is heterogeneous and upstream discharge is a priori unknown. Here we propose a practical solution : it consists in representing separately the debris flow body using a similar fluid (fine mud), and the front by dispersing coarse particles upon the channel bed. These particles are dragged and pushed by the flood so that they finally form a front with global characteristics analogous to those of natural fronts (larger yield stress than the rest of the fluid, unstable behaviour). Then, analyzing our experimental results concerning such flows, we discuss the conditions to respect for a global similarity. 
similitude physique la plus complète possible, nous analyserons et simplifierons au mieux le problème. Nous verrons aussi comment d'un point de vue théorique obtenir une similitude correcte sur certains points. Dans la seconde partie nous synthétiserons des résultats d'expériences préliminaires destinées à comprendre les possibilités de modélisation sur modèle réduit. Enfin dans la dernière partie on développera une méthode pratique pour obtenir un modèle réduit le plus pertinent possible.

\section{Analyse du problème}

\section{I. Généralités}

Pour reproduire l'évolution physique d'un système à une autre échelle il faut respecter des règles de similitude. Ces règles, qui établissent un lien entre les paramètres physiques du modèle et du prototype, sont établies en général a partir des équations qui régissent le système. Le préalable indispensable à l'établissement de lois de similitude est donc la détermination des lois physiques prédominantes pour l'évolution du système qui nous intéresse en l'occurence le mouvement du matériau dans le lit du torrent et éventuellement sur le cône de déjection. Les lois physiques prédominantes dépendent de la facon dont on a cerné le problème. Ainsi le parti pris de Davies [9] est de considérer qu'il faut, pour représenter les laves granulaires, utiliser un mélange d'un fluide interstitiel et de grains, en conservant la similitude de viscosité du fluide et de densité des grains. Malheureusement, même si, par hasard ou grâce à l'intuition de l'expérimentateur, cette approche peut s'avérer a posteriori pertinente, il est difficile de le démontrer complètement. Notamment, dans le cas cité plus haut, il serait nécessaire de prouver que tout se passe comme si les mouvements de l'ensemble du matériau réel étaient effectivement régis par ceux d'un fluide interstitiel et de grains. Enfin l'approche qui consisterait quant à elle à essayer de représenter en similitude tous les phénomènes physiques se produisant au niveau de la microstructure s'avère irréalisable en pratique. En effet, pour mettre au point une similitude fondée sur ce principe on devrait utiliser un fluide modèle au sein duquel tous les types d'interactions entre particules se produisant au cours du mouvement reproduiraient en similitude ceux se produisant au sein du fluide réel. Pour parvenir à un résultat correct il est préférable d'envisager le problème différemment. Notamment on considérera que les évolutions physiques du système qui nous intéressent correspondent à des évolutions mécaniques globales. Dans ces conditions on se placera autant que possible dans le cadre de la mécanique des milieux continus et les problèmes de similitude devront être envisagés de ce point de vue. Pour les phénomènes qui ne s'y rattachent pas a priori (mouvement ou formation du front, érosion,...) on sera amené à comprendre et reproduire en similitude la physique du phénomène.

Comme il a été montré précédemment [1-2] il est possible de considérer en première approximation que l'écoulement d'une lave torrentielle se présente sous la forme suivante: un front très granulaire suivi d'un corps à peu près homogène au volume beaucoup plus important puis de la queue de lave, beaucoup moins visqueuse et dont on négligera l'effet. Le milieu constitutif du corps des «laves torrentielles boueuses " a fait l'objet de diverses études [2] qui ont montré qu'il s'agit d'un fluide dont la loi de comportement peut être approximée par un modèle du type HerschelBulkley. Les laves torrentielles granulaires, quant à elles, devront faire l'objet d'études plus approfondies. Nous n'envisagerons dans la suite que le cas des laves essentiellement boueuses, c'est-à-dire celles qui contiennent une fraction argileuse supérieure à environ $10 \%$. Le front de ces laves a un comportement qui peut vraisemblablement être représenté par une loi de comportement possédant un minimum [2]. La lave torrentielle boueuse qui nous intéresse ici est donc constituée de deux matériaux différents s'écoulant l'un derrière l'autre. Le matériau du corps est un fluide dont la loi de comportement est bien maitrisée. Les équations qui régissent son mouvement sont donc connues et on verra dans la suite (1.2) qu'il est possible alors d'établir des lois de similitude complètes pour ce qui concerne son mouvement. La situation est moins claire pour le front et pour la condition à l'interface entre le front et le corps. Finalement, pour un modèle réduit, la meilleure solution nous semble être d'utiliser un fluide représentant en similitude correcte (du point de vue de la mécanique des fluides) le corps des laves torrentielles boueuses et de reproduire (autant que possible en similitude avec le phénomène naturel) les conséquences de l'existence du front sur l'écoulement de l'ensemble.

On notera que le rôle du front sur l'écoulement ou les débordements ne peut pas être négligé a priori (freinage, blocage dans des rétrécissements, ...), mais son rôle sur l'extension de la lave sur le cône de déjection est faible car son volume est faible. Dans un modèle réduit on devra donc tenir compte du front essentiellement pour étudier des écoulements canalisés.

\subsection{Détermination du fluide modèle pour représenter le corps de lave}

Le problème à résoudre ici est la détermination d'un fluide modèle qui permet de représenter en similitude les écoulements quelconques du corps des laves boueuses. Considérons l'écoulement d'un fluide ayant une loi de comportement du type Herschel-Bulkley [8]. Cet écoulement est régi par les trois nombres adimensionnels suivants:

$F=\sqrt{\frac{V^{2}}{g L(\cos i)}} ; \quad G=\frac{\rho g L(\sin i)}{\tau_{c}} ; \quad H_{b}=\frac{\tau_{c}}{K}\left(\frac{L}{V}\right) n$

où $L$ est une longueur de référence, $i$ la pente du canal, $g$ la pesanteur, $\rho$ la densité, $V$ une vitesse de référence, $\tau_{\mathrm{c}}$ le seuil de contrainte du matériau et $K$ un paramètre du matériau.

- $G$ donne une idée du rapport entre la contrainte à la paroi et le seuil de contrainte du fluide ;

$-H_{b}$ donne une idée du rapport du seuil de contrainte et 
de la partie des contraintes qui varie avec l'intensité du cisaillement.

Le respect de la similitude entre un écoulement prototype et le modèle réduit impose le respect de l'égalité de ces nombres adimensionnels du prototype et de la réalité ainsi que la similitude des conditions initiales et des conditions aux limites. Nous ne sommes concernés ici que par la première condition. Voyons quelles sont les contraintes qui en découlent. On suppose qu'une lave torrentielle naturelle (à l'échelle $L_{1}$ ) de densité $\rho_{1}$ suit une loi de comportement du type Herschel-Bulkley de paramètres $K_{1}, n_{1}$, et $\tau_{\mathrm{cl}}$. Le modèle réduit (à l'échelle $L_{2}$ ) est réalisé dans le même champ de pesanteur avec un fluide de densité $\rho_{2}$ et dont les paramètres de la loi de comportement sont $K_{2}, n_{2}$, et $\tau_{\mathrm{c} 2}$. En premier lieu il est nécessaire que $n_{1}=n_{2}=n$. En fait il s'avère que pour la plupart des suspensions naturelles concentrées, on peut prendre $n=1 / 3$ [2], hypothèse que l'on conserve pour ces deux fluides. Posons $\frac{L_{2}}{L_{1}}=\lambda$. Alors
il faut que :

* $\frac{V_{1}^{2}}{g L_{1}}=\frac{V_{2}^{2}}{g L_{2}}$ ce qui nous donne $: \frac{V_{2}}{V_{1}}=\sqrt{\lambda}$

* $\frac{\rho_{1} g L_{1}}{\tau_{c 1}}=\frac{\rho_{2} g L_{2}}{\tau_{c 2}}$ ce qui nous donne : $\tau_{c 2}=\tau_{c 1} \frac{\rho_{2}}{\rho_{1}} \lambda$

* $\frac{\tau_{c 1} L_{1}^{n}}{K_{1} V_{1}^{n}}=\frac{\tau_{c 2} L_{2}^{n}}{K_{2} V_{2}^{n}}$ ce qui nous donne : $K_{2}=K_{1} \frac{\rho_{2}}{\rho_{1}} \lambda^{7 / 6}$.

On a aussi $Q_{2}=Q_{1} \lambda^{5 / 2}$ et $T_{2}=\sqrt{\lambda} T_{1}\left(Q_{i=1,2}\right.$ et $T_{\mathrm{i}=1,2}$ sont des débits et des temps correspondants dans le modèle et la réalité). Cette similitude théorique conduit en pratique à réduire les paramètres $K$ et $\tau_{\mathrm{c}}$. Si $\lambda$ n'est pas trop petit, le rapport de réduction est le même pour les deux paramètres. On peut alors utiliser un mélange boueux fin dont les paramètres de la loi de comportement seront ajustés en réglant la concentration solide à un niveau adéquat. En général, ne connaissant pas avec précision la valeur des paramètres rhéologiques de la lave torrentielle réelle, on fera des essais dans une gamme de variation plus ou moins large de ces paramètres ( $\mathrm{cf} 3.2$ ). Ceci permettra de s'affranchir du problème posé pour réaliser une similitude exacte lorsque $\lambda$ est petit car on fera en sorte que le rhéogramme des fluides "idéaux " soit compris dans l'enveloppe des rhéogrammes des fluides effectivement testés.

\subsection{Similitude du front}

Comme on l'a dit plus haut le front peut être considéré comme un fluide granulaire, matériau dont la loi de comportement est encore trop mal maîtrisée à ce jour. En outre on ne sait pas encore bien comment il se forme dans les écoulements naturels. Il est vraisemblable qu'un phénomène complexe se produit : l'érosion du lit est particulièrement importante au niveau du front de la coulée, les gros blocs ont tendance à rester plus longtemps que le reste du matériau près du front car ils ont une vitesse moyenne plus grande [1], le front rocailleux forme parfois un véritable mur (description courante de la part des observa- teurs) qui doit freiner le reste de la coulée et se faire pousser. Par ailleurs tout au long de son mouvement dans le lit du torrent, la lave laisse des dépôts latéraux contenant des blocs d'un diamètre relativement élevé. Bien que la lave abandonne ainsi continuellement des gros blocs, le front existe toujours. Enfin il n'est pas envisageable que l'acheminement des gros blocs vers le front soit suffisamment rapide pour être prédominant dans la constitution du front (le rapport entre la vitesse maximum des blocs et la vitesse de la coulée est trop proche de 1). Toutes ces réflexions appuient l'idée que l'érosion de gros blocs à l'avant de la coulée est un facteur prédominant pour l'existence et la formation du front.

Ainsi donc le front trouve son existence dans des phénomènes complexes qui ne peuvent pas encore être mis en équations. De plus, les conditions à l'interface entre le front et le reste de la coulée sont évidemment difficiles à cerner. On ne peut pas imaginer de reproduire le front directement par un fluide granulaire car on ne connaît pas les règles de similitude qui doivent être respectées dans ce cas. En revanche, s'appuyant sur les considérations du paragraphe précédent, il paraît possible de représenter le mode de formation du front. Si les conditions de similitude concernant les différents matériaux en jeu (en l'occurrence les blocs et le corps de la lave) et si les conditions initiales sont respectées, on peut alors affirmer qu'il existera une similitude approchée entre les écoulements naturel et réduit.

Pour parvenir à la création d'une lave formée d'un front et d'un corps en similitude avec la lave naturelle il suffirait donc de reproduire correctement l'érosion du lit. Pour cela la méthode que nous proposons consiste à répartir sur le fond du canal des blocs de densité telle que le rapport (densité des blocs/densité moyenne du corps de lave) est le même dans la nature et sur le modèle réduit. La taille de ces blocs n'est pas nécessairement en similitude géométrique avec les blocs formant le front puisque c'est essentiellement le mécanisme de formation du front que l'on cherche à reproduire. Cependant il convient de déterminer les dimensions et la répartition de ces blocs nécessaires pour obtenir le phénomène souhaité ( $\mathrm{cf} . \mathrm{Ch} 2$ ). On enverra une bouffée du mélange boueux (déterminé en 1.2) par exemple en ouvrant brutalement un barrage placé à l'amont du canal. Comme on le verra dans le chapitre suivant, un front se forme alors. Cependant les conditions expérimentales et le lien avec la réalité doivent être examinés plus précisément.

\section{Expériences}

\subsection{Procédure expérimentale}

De facon à mieux comprendre et vérifier les mécanismes qualitativement décrits plus haut et pour cerner les possibilités réelles d'étude sur modèle réduit des laves torrentielles boueuses, nous avons réalisé des essais sur le principe proposé au chapitre précédent. Nous avons utilisé un canal de $11,5 \mathrm{~m}$ de longueur, de pente $10 \%$, et de section approximativement rectangulaire de $19 \mathrm{~cm}$ de large sur $5 \mathrm{~cm}$ de haut. Lorsque le niveau de fluide dépassait cette 
cote il lui était possible de déborder sur les côtés qui étaient au même niveau que le haut des bords du canal. Ce phénomène est cependant resté mineur pendant nos expériences. Des seuils étaient répartis dans le canal tous les $25 \mathrm{~cm}$ sur une distance de $5,5 \mathrm{~m}$. Ces seuils représentaient approximativement des barrages de $1,5 \mathrm{~cm}$ de hauteur (par rapport au fond du canal) et de $1 \mathrm{~cm}$ d'épaisseur. Le canal était alimenté par l'intermédiaire d'un barrage muni d'une vanne mobile verticalement. La section du barrage était trapézoïdale (hauteur $33 \mathrm{~cm}$; base $19 \mathrm{~cm}$; pente des bords $33 \%$ ). Les côtés de ce barrage se prolongeaient vers l'aval $50 \mathrm{~cm}$ après la vanne, de facon à éviter des débordements lors d'une ouverture brutale. Nous avons choisi de lâcher systématiquement le même volume de 901 , ce qui correspondait à une hauteur de fluide stockée derrière le barrage de $26 \mathrm{~cm}$. Les blocs répartis sur le fond étaient fabriqués en béton léger et avaient une masse volumique moyenne de $1700 \mathrm{~kg} / \mathrm{m}^{3}$. Des blocs de deux diamètres moyens différents ont été utilisés : 3 et $6 \mathrm{~cm}$. Le fluide boueux en similitude du corps d'une lave torrentielle était un mélange eau-kaolinite dont le seuil de contrainte était compris entre 12 et $13,5 \mathrm{~Pa}$.

Dans la suite on décrira les résultats expérimentaux de manière essentiellement qualitative. Les seules mesures qui ont été prises sont les temps de parcours de chaque coulée, c'est-à-dire le temps mis par le front de la coulée pour parvenir à l'extrémité du canal ou le temps mis par ce front avant d'être brutalement freiné (dans tous nos essais juste avant l'extrémité du canal). Les différentes coulées ont été filmées. Après chaque test on a apprécié qualitativement les résultats visibles dans le canal. Le volume dans le barrage était ajusté pour que la lave s'arrête avant l'extrémité aval du canal si le front s'était correctement formé (avec tous les blocs ou presque) après une ouverture brusque complète du barrage amont.

\subsection{Résultats}

Lorsqu'aucun bloc n'était placé dans le canal, une ouverture brusque du barrage donnait lieu à une vague de fluide atteignant l'extrémité aval du canal en $8 \mathrm{~s}$ (l'étude d'écoulements transitoires de ce type a fait l'objet d'une étude systématique [10]). En revanche lorsque le lit était tapissé de gros blocs $(6 \mathrm{~cm})(4$ blocs $/ \mathrm{m})$ un front constitué de tous les blocs se formait à l'avant et atteignait l'aval du canal au bout de $15 \mathrm{~s}$.

\subsubsection{Lien entre le front et lérosion}

Des expériences identiques ont été réalisées en répartissant le même volume total de blocs sur des distances différentes. Il est apparu clairement que le front se formait correctement et persistait là oủ il pouvait régulièrement s'alimenter par érosion. Dans le cas contraire il se désagrège progressivement, la boue passant progressivement par-dessus les blocs au niveau du front. Ce phénomène montre clairement le rôle de frein de l'érosion et du front sur le reste de la coulée. Sans érosion constante la lave gagne de la vitesse et le front se disperse. Tant que des blocs sont disponibles, le front, en les emportant, se freine et se surélève suffisamment pour maintenir le reste de la boue derrière lui. Si le front devient trop important, des blocs commencent à se déposer sur les bords du canal.

\subsubsection{Lien entre le front et l'érodabilité}

Des expériences ont été réalisées avec différentes quantités de blocs dispersés dans le canal et différentes granulométries ( $3 \mathrm{~cm}, 6 \mathrm{~cm}$ ou $3 \mathrm{et} 6 \mathrm{~cm}$ en proportions égales). Il est apparu que la formation du front et le mouvement de l'ensemble dépend beaucoup du volume total de blocs (répartis ici sur toute la longueur) et donc de l'érodabilité, mais pratiquement pas de la granulométrie (dans notre gamme de tests). Par exemple des tests effectués dans des conditions identiques à celles exposées en introduction de cette section mais avec respectivement 2 blocs $/ \mathrm{m}$ et 8 blocs $/ \mathrm{m}$ ont donné lieu à des coulées qui ont parcouru la quasi-totalité du canal en $8,7 \mathrm{~s}$ et $25 \mathrm{~s}$. Dans les deux cas pourtant, le front final était formé de l'ensemble des blocs disposés dans le canal. Compte-tenu du volume limité de fluide envoyé de l'amont, une trop grande quantité de blocs disposés dans le canal aurait stoppé la coulée.

\subsubsection{Lien entre le front et l'énergie de la vague}

On a réalisé des séries d'expériences identiques avec différentes conditions d'ouverture du barrage. Il est apparu que la formation du front dépendait fortement de l'énergie de la vague. Pour une ouverture initiale de la vanne correspondant à la moitié de la hauteur initiale de fluide, le front se forme de manière identique mais la lave met plus de temps à parcourir le canal. Pour une ouverture de la vanne de $2 \mathrm{~cm}$, le front se forme mal avec les gros blocs $(6 \mathrm{~cm})$ mais plutôt mieux avec les petits blocs. En revanche dans ces deux derniers cas il reste une grande partie des cailloux dans la coulée à l'amont du front.

\section{Suggestions pour l'étude de laves torrentielles boueuses sur modèle réduit}

\subsection{Détermination du volume maximum de lave}

On considère le problème général de l'étude de l'écoulement d'une lave torrentielle dans un canal donné. Cette étude a pour but la construction d'un aménagement particulier ou la délimitation d'une zone à risques. Les conditions aux limites géométriques du problème sont donc données. Les caractéristiques dynamiques de l'écoulement sont en revanche plus difficiles à déterminer. En fait on aura à étudier les conséquences de tous les écoulements possibles de laves torrentielles dans ce canal. Cependant on ne peut pas en pratique tester l'effet de tous les phénomènes qui sont susceptibles de se produire. En effet, compte-tenu des connaissances actuelles dans le domaine du déclenchement des laves torrentielles, il n'est pas possible de connaitre les limites supérieures des phénomènes à envisager dans ce cas. Il semble que des phénomènes imprévisibles et d'une ampleur exceptionnelle, dont les 
hommes ne pourront avoir prévu de se protéger, soient toujours à craindre. Dans ces conditions, on se basera sur les phénomènes classiquement observés dans le torrent qui nous intéresse. On se fixera donc finalement un volume de lave qui sera établi à partir de données antérieures concernant ce torrent et correspondra à peu près au plus grand volume que l'on peut craindre en restant dans des limites raisonnables. Après cette phase dont le caractère politique et social ne doit pas être négligé, il ne reste plus qu'à déterminer les conditions initiales de l'écoulement et le fluide modèle à utiliser.

\subsection{Détermination du fluide modèle}

$\mathrm{Si}$ on possède des échantillons de lave prélevés dans le torrent étudié juste après une coulée naturelle, il est possible d'estimer le seuil de contrainte de la lave complète relativement précisément. Ceci peut se faire en multipliant le seuil estimé de l'échantillon partiel (en général contenant les particules de diamètre inférieur à quelques centimètres) par 1,5. Une technique pratique complète d'estimation du seuil de la lave complète est proposée dans [11]. On obtient ainsi une estimation du seuil réel à environ $35 \%$ près. On testera alors sur le modèle réduit des laves ayant un seuil compris dans une gamme encadrant la valeur obtenue ci-dessus. Si on ne possède pas d'échantillons "frais " de lave torrentielle, nous suggérons de considérer que la lave a un seuil de contrainte compris entre 500 et $1500 \mathrm{~Pa}$ car cette gamme semble encadrer la plupart des seuils observés sur le terrain.

Le fluide modèle sera choisi en respectant la règle de similitude concernant le seuil de contrainte (cf. 1.2). On utilisera préférentiellement un mélange eau-argile naturelle. Comme toutes les suspensions de ce type ont une loi de comportement qui peut être bien représentée par un modèle du type Herschel-Bulkley avec un paramètre $n$ proche de $1 / 3$, et comme on est de toute facon amené à considérer une gamme de comportement, on ne se souciera pas d'avoir une similitude exacte concernant le paramètre $K$ (cf. 1.2). Enfin on notera que ce mode de détermination du fluide modèle ne requiert en aucun cas le respect de la similitude géométrique de la granulométrie. Seules des considérations concernant des phénomènes particuliers dus à la présence de ces particules dans la coulée justifieraient cela.

\subsection{Détermination des conditions initiales}

Outre le problème pratique de la représentation du front, se pose ici le problème plus général de la représentation en similitude des conditions initiales de l'écoulement transitoire de boue à l'amont du canal (cf. 1.3). D'après les observations de terrain il semble que les laves aient toujours des caractéristiques proches. Ceci est probablement dû au fait qu'un équilibre progressif s'établit dans le lit du torrent entre le front, la coulée et l'érosion du lit. D'une part le volume du front ou son seuil de contrainte ne peuvent pas devenir trop importants sinon la lave s'arrête. D'autre part, si le front se désagrège la boue coule avec un niveau plus faible et une capacité érosive plus faible, au point que la lave dans son ensemble se désagrège. On peut en conclure qu'une lave qui s'écoule effectivement doit avoir des caractéristiques comprises dans une gamme relativement étroite. Dans ces conditions, l'idéal serait de pouvoir reproduire sur modèle réduit le processus dans son ensemble. Malheureusement cela n'est pas possible parce qu'on ne dispose pas en général d'une installation de longueur suffisante.

On cherche donc à reproduire le phénomène comme décrit dans la section 1. Pour cela on utilisera des blocs en similitude de densité correspondant à des blocs réels d'un diamètre de l'ordre du mètre et répartis sur le fond du canal. Ces blocs ne sont pas nécessairement en similitude géométrique avec les blocs réels puisqu'on cherche seulement à reproduire le phénomène de front. Cependant lorsqu'on cherche à étudier les effets de différents ouvrages sur des laves torrentielles il est important de tenir compte de la taille des blocs réels. Il paraît délicat de représenter en similitude l'ensemble de la granulométrie des grains compris dans le front. En effet, celle-ci est très étendue et variable d'une lave à l'autre. Dans ce cas, la comparaison de l'effet des différents ouvrages pourra être faite en effectuant des tests identiques avec un front donné, puis éventuellement en faisant varier la granulométrie du front. Pour préciser les conditions d'essais systématiques il pourra être utile, au cours d'essais préliminaires, d'avoir fait varier la densité de répartition de ces blocs sur le canal.

Le problème clé est maintenant de déterminer les conditions initiales de l'écoulement transitoire de boue. On propose donc de chercher à recréer le réglage naturel de l'intensité de la vague. Pour cela on va chercher à atténuer la vague que l'on créera à l'amont par l'intermédiaire de l'écoulement en lui-même. Une solution pourrait être de lâcher les vagues à l'amont d'un serpentin situé lui-même à l'amont du canal étudié.

En parallèle d'une étude à partir de ces techniques on pourra aussi étudier les conséquences de certains de ces aménagements à partir d'écoulements de laves en régime permanent. La difficulté reste alors de déterminer le débit à imposer. Cependant s'il s'agit seulement de comparer les effets de différents aménagements, il est raisonnable de travailler sur un seul débit, correspondant par exemple à un débit de plein bord dans une zone donnée.

\section{Conclusion}

Nous avons abordé un problème extrêmement complexe. De nombreux phénomènes qui y sont rattachés sont encore actuellement mal compris. Les solutions pratiques que nous proposons comportent évidemment une large part d'approximation mais elles tentent de se rattacher au mieux à la physique des phénomènes. Des études futures, notamment celles relatives aux lois de comportement des milieux granulaires permettront de progresser dans cette voie. Cependant il nous semble que seuls des progrès très sensibles dans la description et la compréhension des phénomènes torrentiels pourraient permettre de modifier sérieusement les méthodes proposées ci-dessus. 


\section{Références}

[1] Coussot P. (1992). - Rhéologie des laves torrentielles, thèse de Doctorat de l'Institut National Polytechnique de Grenoble, Grenoble, France, 420 p.

[2] Coussot P.. (1994). - Rhéologie des laves torrentielles, La Houille Blanche (ce numéro).

[3] Torrent du grand Merdaret: Etude sur modèle réduit, NEYRPIC, Laboratoire Dauphinois d'Hydraulique, Grenoble, France, 30 p., (1952).

[4] Etude hydraulique du franchissement du torrent de la Griaz par la voie express Le Fayet-Les Houches - Etude sur modèle réduit -. SOGREAH, Grenoble, France, (1985).

[5] Johnson A.M., (1970). - Physical processes in Geology, Freeman Cooper and Co, $577 \mathrm{p}$.

[6] WARD T.J. and O'BRIEN J.S., (1980). - Flume mechanics of mud flows, Completion report to National Science foundation
Research Initiation, CER80-81TJW-JSO2, Fort Collins, Colorado, U.S.A., $130 \mathrm{p}$.

[7] Takahashi T., (1991), - Debris flow, International Association for Hydraulic Research, Monograph Series, A.A. Balkema, Rotterdam. Netherlands, 165p.

[8] Coussot P.. (1994). - Lois d'écoulement des laves torrentielles, La Houille Blanche (ce numéro).

[9] DAviEs T.R.H. (1993), - Models for disastrous mass movements. Rapid Gravitational Mass Movements, Pierre Beghin International Workshop, Grenoble. France.

[10] Laigle D. et Coussot P., (1993). - Modélisation numérique des écoulements de laves torrentielles, La Houille Blanche (ce numéro).

[11] Coussor P., (1993). - Mèthode de détermination pratique des paramètres rhéologiques des laves torrentielles boueuses, Note Technique, CEMAGREF, Division Protection contre les Erosions. 